\section{ETNOARQUEOLOGÍA DE LA EXTRACCIÓN DEL ORO DE LOS RÍOS EN EL NOROESTE DE LA PENÍNSULA IBÉRICA}

\author{
ETHNOARCHAEOLOGY OF THE \\ EXPLOITATION OF GOLD IN THE \\ RIVERS OF NORTHWEST OF IBERIAN \\ PENINSULA
}

\section{J.M. VÁZQUEZ VARELA (*)}

\section{RESUMEN}

Se presenta el estudio etnoarqueológico de la obtención con técnicas tradicionales del oro fluvial en el curso del río Sil en Galicia. La aplicación del modelo a la joyería prehistórica del Noroeste de la Penísula Ibérica permite pensar la posibilidad de que toda ella fuese hecha con oro de los placeres fluviales usando una técnica de bateado sencillo con una inversión de tiempo y esfuerzo limitada. En consecuencia el valor de ostentación social de las joyas en las zonas ricas en oro pudo ser menor de lo que tradicionalmente se piensa.

\begin{abstract}
An ethnoarchaeological study of the exploitation of fluvial gold with traditional techniques along the Galician Sil river is presented here. The application of the model to prehistoric jewellery in the north-western Iberian Peninsula allows for the possibility that all of it was made of gold, collected from the river basins using a simple technique that neent a limited investment of time and effort. Therefore the valne of the social ostentation of jewels in goldrich areas could be less than was traditionally believed.
\end{abstract}

${ }^{*}$ ) Departamento de Historia 1. Facultade de Xeografía e Historia. Universidade de Santiago de Compostela. 15703 Santiago de Compostela. La Coruña. España.

El artículo fue remitido en su versión final el 5-VI-95.
Palabras clave: Etnoarqueología. Joyería. Oro. Prerromano. Noroeste de España.

Key words: Ethnoarchaeology. Jewellery. Gold. Prerroman. Northwest of Spain.

\section{INTRODUCCIÓN}

Dentro de un programa de investigación etnoarqueológica en Galicia se ha realizado en septiembre de 1994 un estudio sobre las técnicas tradicionales de la recogida del oro del río Sil en la aldea de Pumares, ayuntamiento de $\mathrm{O}$ Barco de Valdeorras en el este de la provincia de Ourense (Vázquez y Rodríguez, e.p.). Su finalidad era conocer una actividad sobre la cual sólo existían pocas y genéricas referencias etnográficas (García, 1974; Sánchez, 1983) y a partir de los datos obtenidos elaborar hipótesis de trabajo aplicables a la interpretación de los aspectos económicos y sociales de la joyería prerromana de la región.

Aquí se presenta una síntesis de los datos etnográficos, descritos con mayor detalle en la publicación citada (Vázquez y Rodríguez, e.p.), su interpretación y aplicación al conocimiento de diferentes cuestiones relativas al aprovechamiento del oro en las comunidades castreñas de cronología avanzada e incluso a la joyería más antigua de la región. 


\section{EL MODELO ETNOARQUEOLÓGICO}

Los datos se recogieron de varios informantes de edad avanzada quienes describieron la práctica de la actividad hace cuarenta años cuando cesó el oficio.

El trabajo de explotación de los placeres fluviales estaba en la mayor parte de los casos en manos de mujeres solteras que trabajaban en grupo durante el verano a lo largo de un tramo, de unos setenta kilómetros aproximadamente. del río Sil, desde los alrededores de Quiroga hasta la provincia de León (Fig. 1).

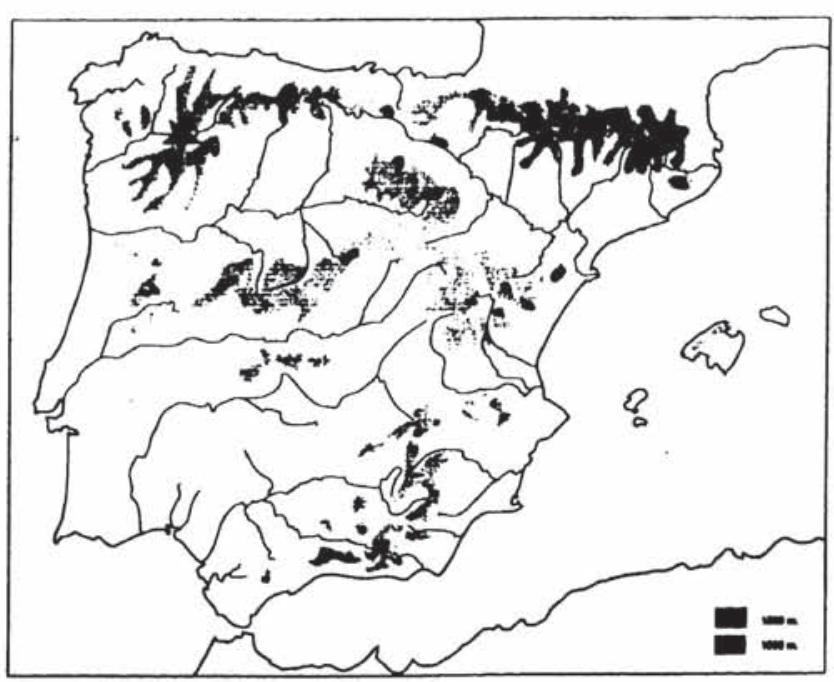

Fig. I. Situación de Pumares en la cuenca del río Sil (Orense).

La dedicación plena al oficio cesaba en el momento del matrimonio, si bien de un modo ocasional, alguna persona cuando disponía de un momento libre en las tareas domésticas o campesinas propias de una casada, cogía la batea y bajaba al río inmediato a la aldea donde trabajaba un rato.

El oficio se practicaba en el pueblo al menos desde el siglo pasado. En el diccionario de Madoz (1845) se cita como una de sus industrias el trabajo en el río para el aprovechamiento del oro. El geólogo G. Schulz $(1835,1838)$ alude a la explotación de las arenas auríferas de la comarca. Otros autores señalan la presencia de «aureanas» en amplias zonas del Sil (Becerro de Bengoa, 1833) y en otros ríos de Galicia. En la provincia de Pontevedra, han trabajado hasta los años cuarenta de nuestro siglo (García, 1974).

Su labor consistía en el aprovechamiento del oro, contenido en el sedimento, que las crecidas del río Sil dejaba sobre sus orillas.

Aquél se cogía con una azada de cortas dimensiones, en pequeñas cantidades que se introducían en un cuenco de madera de castaño con la forma aproximada de cono, de un diámetro máximo de cincuenta centímetros. Sumergido en el agua y en la superficie alternativamente se le imprimía, con ambas manos, una serie de movimientos circulares, de modo que se depositase en su fondo el oro, que inevitablemente iba acompañado de arenas finas y se desprendiese la parte estéril.

La labor se realizaba durante los meses de verano, en jornadas muy largas, desde la mañana a la noche, con un descanso en las horas centrales del día para comer a la orilla del río.

La porción de sedimentos que contenían el oro se concentraba en el fondo del cuenco y se recogía en una lata de conservas. Cuando había una cierta cantidad de material se mezclaba todo en el cuenco con mercurio y se azogaba hasta que la masa de éste tomaba el color dorado del oro. A continuación se colocaba el líquido en un recipiente metálico que se calentaba con brasas de brezo por arriba y por abajo. Como consecuencia de esta operación parte del mercurio se evaporaba y el oro quedaba puro.

La cantidad de oro recogida variaba de un lugar a otro, desde sitios en los que no se obtenía nada en toda una jornada hasta otros, excepcionales, donde en el mismo plazo, en un golpe de suerte se podían obtener hasta 5 gramos. Calculan que por término medio, un día con otro, conseguían unos 2 ó 3 gramos por jornada de trabajo. Noticias verbales indirectas de gentes que aún hoy en día buscan esporádicamente oro en zonas cercanas, como Salas de la Ribera, próximo a Pumares, o en Carucedo, en el oeste de la provincia de León, confirman rendimientos equivalentes, en torno a 2 ó 2,5 gramos por jornada de trabajo.

No recuerdan ningún hallazgo excepcional por su cantidad, ni a nadie que se hubiese enriquecido con este trabajo.

Algunas «aureanas», a veces, guardaban algo de oro para hacerse una sortija, pulsera o pendientes para ellas o para sus hijos, pero lo habitual era la venta íntegra de lo obtenido, por ser necesario el dinero para la economía doméstica. 
Llama la atención que el grupo familiar doméstico pudiese prescindir de brazos jóvenes para dedicarlos a este trabajo, en una época del año en que aparentemente todos son necesarios para las faenas agrícolas, particularmente variadas y complejas en la zona por su rico policultivo.

Las entrevistadas explican esta aparente contradicción diciendo que, debido al tamaño de las familias y lo reducido de las fincas pues en la zona se practica el sistema de la herencia a partes iguales, siempre había personas dispuestas para estas tareas.

El oficio de "aureana" puede ser entendido como una estrategia de adaptación al medio en una sociedad que, a pesar de estar en un territorio potencialmente rico, no sale de la pobreza a causa del tamaño de la población, y del sistema de transmisión de la propiedad en las herencias, que condenan a ésta a una división perpetua. La aportación de las mujeres solteras a la comunidad doméstica era apreciada en este tipo de economía, en el que la disponibilidad de dinero contante y sonante era escasa.

Posiblemente el que no fuese una actividad muy rentable explica que se dedicasen a ella las solteras, y sólo de un modo excepcional gente apremiada por la necesidad.

La construcción de los embalses dedicados a la producción de energía hidroeléctrica controla la circulación del río, e impide que sus crecidas depositen el sedimento, del que se obtenía el oro, en las orillas. Con este mecanismo desapareció la fuente de este metal y el viejo, al menos bimilenario, oficio de «aureana».

\section{LA APLICACIÓN DEL MODELO}

El proceso descrito de explotación del oro fluvial coincide bien a grandes rasgos con la descripción que hace Estrabón $(3,2,9)$ del aprovechamiento de las arenas fluviales en los ríos del Noroeste. Por ello se podría pensar que lo estudiado por nosotros es una «sobrevivencia», y que por tanto su valor para entender el trabajo del oro en el pasado sería ideal pues habría llegado hasta nuestros días prácticamente intacto un modo de hacer las cosas propio de la Protohistoria.

Este tipo de uso de la información etnoarqueológica no es posible pues, aunque la técnica fuese la misma, el sistema social en cuyo seno opera es distinto y es evidente que hay profundas diferencias entre la cultura de la Galicia de fines del siglo XX y la de hace dos mil años.

Hay dos diferencias importantes entre la técnica actual y la citada por Estrabón: ahora se emplea un cuenco de madera y el mercurio mientras que las poblaciones prerromanas usaban cestos hechos de fibra vegetal y no parecen haber conocido el citado metal. La segunda es quizás más importante que la primera ya que permite concentrar el oro de un modo que no era posible en el pasado por lo cual los rendimientos actuales pueden ser mayores que los de entonces.

Por otra parte, aunque la coincidencia fuese absoluta entre la técnica actual y la descrita por el clásico, no se puede demostrar que no haya habido solución de continuidad entre ambas épocas. Los datos disponibles permiten saber que, en la primera mitad del siglo pasado, la situación era más o menos parecida a la de 1950 en el aspecto técnico, social y económico (Becerro, 1833; Schulz, 1835; 1838, 1992; López, 1985). También se documenta la explotación durante las época medieval y moderna (Sánchez, 1983).

Las condiciones ambientales también han variado ya que la explotación de la misma comarca durante mucho tiempo ha ido agotando los recursos y éstos aunque renovables lo son a unos plazos muy largos pues dependen de las crecidas del río que arrastran materiales erosionados en otro lugar.

$\mathrm{Si}$ bien las limitaciones citadas impiden tomar los datos etnoarqueológicos como una representación lineal del pasado sí tienen un valor indicativo que los hace sumamente útiles a la hora de hacer hipótesis de trabajo. Desde este punto de vista cabe hacer consideraciones de tipo económico y social: el tipo de técnica y su rendimiento, el valor social de las joyas y el origen del oro de la joyería prerromana.

\section{La técnica, su rendimiento y el valor social de las joyas}

El proceso de recuperación del oro de las arenas fluviales sólo emplea el cuenco y prescinde por completo de algún tipo de criba, por otra parte, el procedimiento más generalizado (Sánchez, 1983). Tal vez esta aplicación más sencilla de la técnica esté relacionada con las características del sedimento lavado, que es el que el río abandona sobre las orillas durante las crecidas. 
De acuerdo con los datos recogidos y transmitidos por Schulz (1838) en la primera mitad del siglo pasado se ha calculado (Sánchez, 1983) que, si el rendimiento de las aureanas se extrapola a las buscadoras de oro prerromanas, éstas recogerían por persona un promedio de 12-14 gramos por estación estival de trabajo por lo cual sería necesario el trabajo de 149 de ellas durante una estación para hacer un torques como el de Burela que pesa 1800 gramos aproximadamente. Una "aureana" podría obtener el oro necesario para hacer un par de arracadas como la de Villar de Santos, de 18 gramos, en tres campañas de verano.

Con los datos del modelo etnoarqueológico se ha comprobado que el rendimiento es de 2 a 3 gramos diarios. Si se toma como valor promedio 2,5 gramos de oro por persona por día, la producción del período estival sería de 150 gramos resultado de multiplicar por el número de días laborales, cinco por semana, en tres meses de acuerdo con nuestro sistema de trabajo, que nos daría 60 días útiles. Esta cifra es aproximadamente diez veces mayor, que la calculada por Schulz (1838) en base a informes globales de gentes del país que le comunicaron el valor total de la producción de la comarca, cosa muy difícil de fiscalizar a nuestro entender. Con nuestro módulo la arracada de Vilar de Santos de 18 gramos pudo ser hecha con el trabajo de una persona en semana y media y el par en tres semanas, y el torques de Burela, el más grande de los gallegos, 1800 gramos, con el trabajo de 12 mujeres en un verano.

El peso de los torques de oro de la región oscila entre el máximo del citado y los 219 gramos del menor. La mayoría de los ejemplares se aproximan a los 500 gramos (Pérez, 1989). Uno de éstos requeriría aproximadamente el trabajo de 3 personas durante un verano. En el caso de la orfebrería más antigua de la región el esfuerzo sería menor ya que las piezas, por lo general, son de peso más reducido (Vázquez, 1993).

Estas nuevas cifras dan una imagen del esfuerzo social que encierran las joyas más moderado que el derivado de los datos de Schulz. Como consecuencia de ello queda clara la posibilidad de que, en las zonas donde había oro fluvial, estuviera al alcance de cualquiera el hacerse con la cantidad necesaria para una arracada en una corta temporada de trabajo y que las piezas de gran envergadura representen la exhibición de un poder social que permitió la acumulación de un esfuerzo laboral más amplio pero no excepcional.

De acuerdo con estos datos parece que a las joyas de la época procedentes de las zonas ricas en oro fluvial ha de concedérseles menos valor indicativo de alto estatus del que se les viene otorgando tradicionalmente sobre la base de la dificultad para la obtención del oro.

Es discutible que las cifras estimadas por nosotros sean idénticas a las de la época prerromana por cuanto no se ha documentado el uso del mercurio que permite rendimientos más altos y más cómodos, con menos trabajo e inversión de energía, sobre todo cuando se trabaja sobre partículas finas de oro como es el caso de las que se encuentran en el limo depositado por el río en sus orillas durante las crecidas. Sin el empleo del citado metal sería necesaria una labor más prolongada para obtener la misma cantidad de oro, pero también se puede considerar que su concentración podía ser más elevada ya que las «aureanas» de ahora trabajan sobre lo que dejaron los anteriores explotadores del río más lo que éste ha ido aportando desde entonces. Las noticias de los autores citados del siglo pasado mencionan también el hallazgo de alguna pepita de oro que daba alegría a quienes la hallaban pero ninguno de los cuales salía de pobre, de lo que se puede deducir que también utilizaban el sedimento del fondo del río. En este caso al explotarse una fracción más gruesa no sería tan importante el empleo del mercurio.

\section{El origen del oro de la joyería prerromana}

El relativo buen rendimiento y facilidad del trabajo en los placeres fluviales documentado por el modelo etnoarqueológico favorece la tesis de Sánchez Palencia (1983) de que posiblemente en el mundo prerromano del Noroeste sólo hubo explotación de placeres fluviales ya que su rendimiento era suficiente para la realización de la joyería de la época. Su afirmación basada en los datos de Schulz (1838) se refuerza con los cálculos etnoarqueológicos que multiplican por diez la supuesta capacidad productiva de la técnica empleada en la época prerromana en el Noroeste de la Península Ibérica.

Para confirmar esta hipótesis es necesario hacer una investigación de tipo geoquímico que, mediante los análisis de las piezas, pueda indicar con gran precisión su origen fluvial o no frente al actual estado de la cuestión que no permite solucionar el tema con toda la seguridad deseable 
(Sánchez y Pérez, 1989). Dado que algunas "aureanas" conservan algo del oro sacado del río, a título de recuerdo, y algunas joyas hechas con él es posible analizar materia prima y piezas para conocer sus contenidos en impurezas y así establecer con más precisión el origen del oro de las piezas prehistóricas y el esfuerzo técnico, económico y social que representan.

La sencilla técnica descrita de recuperación de los placeres fluviales y especialmente los rendimientos calculados han de ser tenidos en cuenta a la hora de valorar la función social de las joyas de oro y su relación con el poder. De lo contrario se corre el riesgo de reconstruir el pasado a nuestra imagen y semejanza por la proyección de los valores del Homo economicus de nuestro tiempo. El modelo etnoarqueológico permite contrastar esta visión actualista y comprender que, en sociedades distintas y con visiones diferentes de lo económico, las cosas han podido ser de un modo distinto al nuestro.

Una joya de tamaño medio puede ser el resultado del trabajo de una persona o de un grupo reducido durante un período de tiempo no muy dilatado, días o quizás meses. No tiene por qué suponer un despliegue de poder económico y social sino que puede tener una lectura más doméstica y ser considerada como el fruto de algo accesible a un mayor grupo de personas en un terreno donde el oro era de fácil recogida y suficiente para las necesidades de la población.

El desarrollo de investigaciones etnoarqueológicas actualmente en marcha sobre diferentes aspectos de la metalurgia puede ayudar a la elaboración de hipótesis que nos permitan nuevas y más fundamentadas lecturas del pasado.

\section{BIBLIOGRAFÍA}

Be(ERRo de Beng(ion. R. (188.3): "De Palencia a La Coruña. Palencia.

Garcia Martinfz. M.C. (1974): “Aurcana”. (iran Enciclopedia Gallega. 3. Silverio (añada Editor. Santiago de Compostela: 19-20.

López Caneda. R. (1985): «El Arciprestazgo de Valdeorras 180()-1825. Religión, Población, Economian. Instituto de Estudios Valdeorrenses. Cuaderno Monográfico, 4. Barco de Valdeorras.

MADOZ. P. (1845): "Diccionario Geografico-EstadisticoHistórico de España y sus posesiones de Ultramar. Galicia". Madrid. Tomo V: 1093.

Perez Outeiriño. B. (1989): “Orfebrería Castreña». En "El oro en la España Prerromana". Revista de Arqueologia: 90-106. Madrid.

SaNChez Palencia. F.J. (1983): «La explotación prerromana del oro del Noroeste de la Península lbérica», Boletin Auriense, XIII: 31-67. Orense.

Sanchez Palencia, F.J y Pérez. L.C. (1989): «Los yacimientos auríferos de la Península Ibérica. Posibilidades de explotación en la Antigüedad». En «El oro en la España Prerromana». Revista de Arqueología: 16-23. Madrid.

Schulz. G. (1835): “Descripción Geognóstica del Reino de Galicia”. Imprenta de los Herederos de Collado. Madrid.

- 1838): «Ojeada sobre el estado actual de la minería en el distrito de Asturias y Galicia». Anales de Minas, 1: 379-397.

- (1992): “Cuadernos de campo, $n^{\circ}$ 2. (Mayo-Agosto 183.3)». Edición de J.R. Vidal Romaní. Edicións do Castro. La Coruña.

VAzouez VAREla, J.M. (1994): «Arte Prehistórico». En "Galicia Arte», IX, Arte Prehistórico y Romano. Hércules de Ediciones. La Coruña.

VAzouez Varela. J.M. y Rodriguez Martínez, M.M. M. (e.p): «Las mujeres del oro: "Aureanas" de Pumares, Ourense (Galicia)». Trevaruna, III, 1994. Castelo Branco. 